\title{
Aplicando las TIC en el aprendizaje de la enfermería profesional
}

\author{
Guadalupe Nohemí López Colmenares
}

\begin{abstract}
Resumen
Las tecnologías de la información y la comunicación (TIC) han incursionado en el ámbito educativo como herramientas auxiliares dentro del proceso enseñanza-aprendizaje. Estudios previos muestran que las TIC son mayormente utilizadas para la comunicación y que existe un desuso en el ámbito educativo. Se realizó un estudió exploratorio, donde participaron 58 estudiantes de la carrera en enfermería, quienes tuvieron acceso a un blog como TIC auxiliar para el aprendizaje durante la práctica clínica. Se aplicó el instrumento UTAE-1 (Uso de TIC para el aprendizaje en estudiantes), que midió el grado de conocimiento y dificultad ante el uso de TIC para el aprendizaje; así mismo, se evaluó el rendimiento académico antes y después de utilizar el blog. 74\% de estudiantes fueron mujeres de edad promedio entre 21 y 23 años. No se encontró un efecto en el rendimiento académico de los alumnos después de usar el blog como auxiliar para el aprendizaje. Las TIC en la enseñanza actual no son la panacea en un ambiente de aprendizaje, más son un auxiliar en donde los alumnos pueden usar sus habilidades tecnológicas en beneficio de su formación profesional. Se propone explorar de manera cualitativa el efecto en el alumno al utilizar las TIC como auxiliar para el aprendizaje.
\end{abstract}

Palabras clave: TIC para el aprendizaje, aprendizaje en enfermería, rendimiento académico.

\section{APPLYING ICT IN THE LEARNING OF PROFESSIONAL NURSING}

\begin{abstract}
Information and Communication Technologies (ICT) have entered the educational field as auxiliary tools in the teaching-learning process. Previous studies show that ICT are mostly used for communication and that there is disuse in the educational field. An exploratory study was conducted; 58 nursing career students, with access to a blog as ICT assistant for learning during clinical practice, participated. UTAE-1 instrument (Use of ICT for Student Learning) was applied; it measured the degree of knowledge and difficulty in the use of ICT for learning, and academic performance was assessed before and after using the blog. 74\% of students were women of age average between 21 and 23 years. There was no effect on students' academic performance after using the blog as an aid for learning. ICT in current education are not the panacea in a learning environment, but they are auxiliary, a place where students can use their technological skills in benefit of their professional training. We propose to explore qualitatively the effect of ICT as an aid for learning on students.
\end{abstract}

Keywords: IcT for learning, nursing learning, academic performance.

DOI: http://doi.org/10.22201/codeic.16076079e.2019.v20n5.a8 


\section{Guadalupe Nohemí López Colmenares}

lopezg720@hotmail.com

https://orcid.org/0000-0002-5328-644X

Licenciada en Enfermería por la Facultad de Estudios Superiores Zaragoza y maestra en Enfermería por Unidad de Posgrado de la Universidad Nacional Autónoma de México (UNAM).

Estudiante del doctorado en Educación por la Universidad Marista, Colegio Universitario de México. Profesora de asignatura de la carrera de Enfermería, Facultad de Estudios Superiores Zaragoza, UNAM.

Sus temas de Investigación son la educación en enfermería, evaluación curricular en enfermería y la innovación en el cuidado de enfermería.

\section{Introducción}

Existen antecedentes que mencionan la importancia del uso de las tecnologías de la información y la comunicación (TIC) por parte de los estudiantes para hacer significativo su aprendizaje y mejorar el rendimiento académico, además de la importancia que tiene la intervención innovadora del docente durante el proceso formativo. En el momento de buscar las causas del fracaso escolar - bajo rendimiento académico, los programas de estudio, la masificación de las aulas y el uso de metodologías no innovadoras se convierten en factores predisponentes.

Por su parte, los profesores en la búsqueda de solucionar el problema se preocupan por desarrollar la inclusión de las Tic como un auxiliar innovador para la enseñanza; sin embargo, se ha observado cierta apatía por parte de los estudiantes para utilizarlas en su aprendizaje.

La educación tiene que adaptarse en todo momento a los cambios de la sociedad. Mientras la sociedad de la información se desarrolla y multiplica, la educación debe permitir que todos puedan aprovechar esta información; recabarla, seleccionarla, ordenarla, manejarla y utilizarla (Peñalosa, 2013).

El uso de internet como recurso para la consulta de información es el de mayor recurrencia (61.9\%), las actividades relacionadas con la comunicación personal (correo electrónico, chat, etcétera) tienen una proporción de 60.9\%, mientras que el uso con fines de apoyo a las actividades escolares se menciona con un 31.6\% (INEGI, 2012).

Manejar las tic para el aprendizaje involucra de manera activa tanto a los alumnos como a los profesores. Por un lado, los profesores deben de innovar en las metodologías de enseñanza para poder llegar a la construcción de un aprendizaje significativo en los alumnos, mientras que éstos deben mantener un interés en lo que desean aprender. 
La selección adecuada (de acuerdo al contexto social y cultural de los alumnos y necesidades del ambiente de aprendizaje) de una tic puede favorecer su utilidad y hacer de ella un auxiliar en el proceso formativo de los estudiantes que permita la asimilación de nuevos conocimientos.

Es de suma importancia poder mantenerse a la vanguardia en cuanto a los nuevos descubrimientos y aportaciones a la sociedad del conocimiento en el área de la salud. En la profesión de enfermería, el cambio de paradigma en el cuidado obliga a mantenerse actualizado para estar en sintonía con los demás profesionales y proporcionar a la persona, individuo y comunidad una atención de calidad.

Dentro de las ciencias de la salud, se realizan múltiples actividades de tipo explicativo o procedimental, por lo que es útil apoyarse en las tic para mejorar los ambientes de aprendizaje, y que, por medio de éstos, en la sección de recursos se envíe material audiovisual que refuerce los conocimientos, optimice tiempos y permita que puedan llevarse a cabo las técnicas exploratorias enseñadas (Díaz, 2010).

La educación requiere que los estudiantes se interesen por su aprendizaje, busquen el logro de sus metas personales y que manifiesten satisfacción por lo que algún día será su profesión (Wison, 1995: 27).

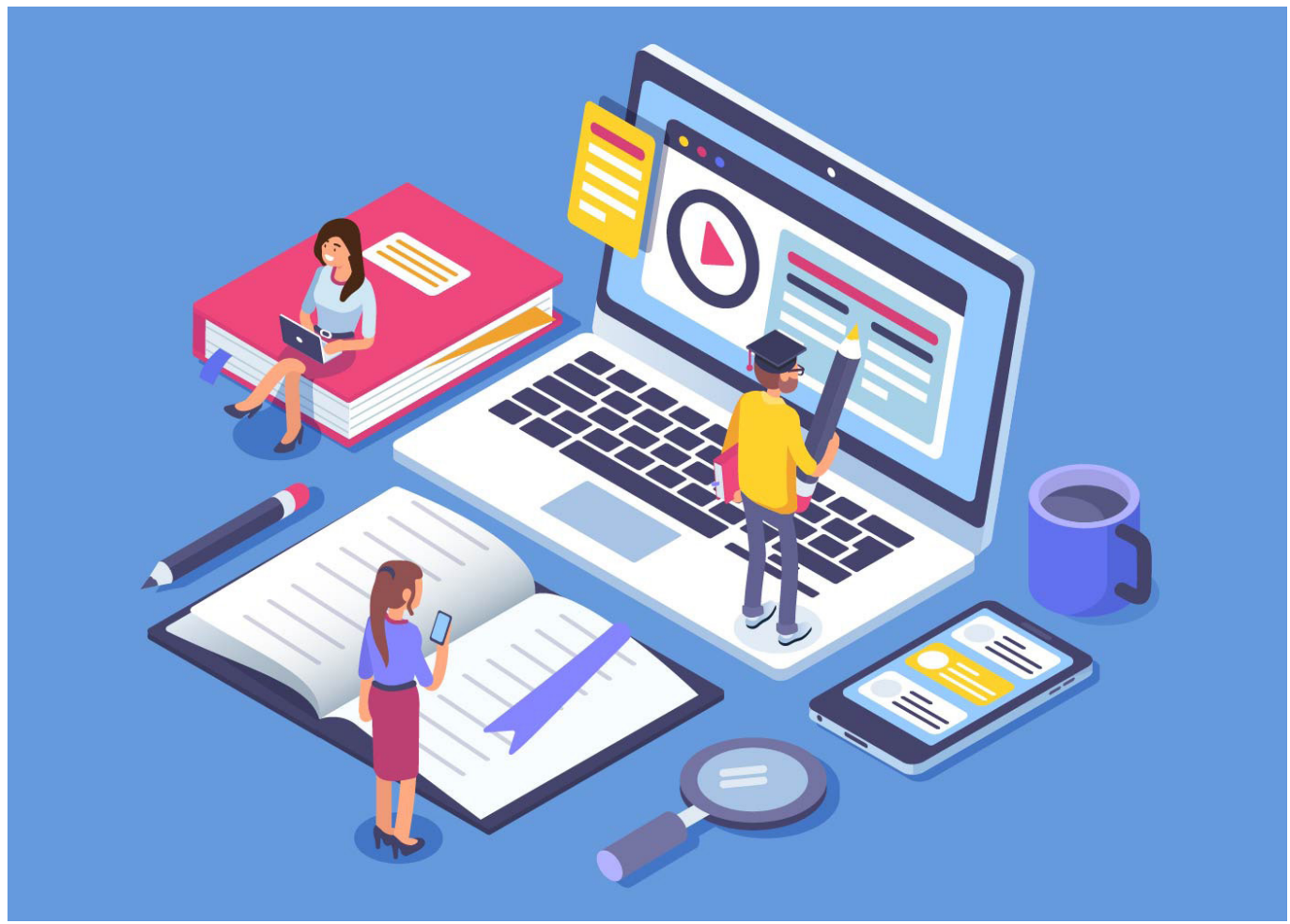




\section{Tipología de herramientas tecnológicas para la educación}

Con el paso del tiempo, las tic han dado grandes saltos de modernización dando paso a una segunda generación de comunidades virtuales basadas en la web. Lo anterior ha permitido la creación de redes sociales, blogs, wikis, entre otros servicios cuyo objetivo se basa en la colaboración y en el compartir contenidos y recursos entre varios usuarios, mediante diversos dispositivos digitales.

Podemos identificar ocho familias de herramientas tecnológicas para la educación (Ley, 2015):

1. Ambientes de aprendizaje

2. Presentación de contenidos

3. Co-construcción colaborativa de contenidos

4. Herramientas de autoría

5. Medios de comunicación

6. Herramientas de inmersión

7. Estrategias de aprendizaje

8. Herramientas cognitivas

Dentro de la categoría de co-construcción colaborativa se encuentran los blogs, definidos como sitios web que se actualizan de manera continua y periódica, que recopilan cronológicamente información (artículos, textos originales, de uno o varios autores).

Para García (2005), los blogs son considerados como "la imprenta personal del siglo xxı". Son espacios de comunicación asíncrona caracterizados por la recopilación de documentos y por la aparición en primer lugar de la aportación más reciente. Al ser utilizados para el aprendizaje son denominados edublogs.

Para los estudiantes, los edublogs son excelentes herramientas que fortalecen su aprendizaje y que permiten una mayor emancipación de los profesores. Les generan un aprendizaje autorregulado. Algunos de los usos que se les puede dar a los edublogs son:

- Publicar materiales o recursos educativos abiertos

- Establecer discusiones en línea

- Crear y compartir trabajos en conjunto

- Colocar archivos multimedia

- Contar con un sitio web del curso

- Organizador de tareas, eventos o prácticas

- Seguimiento de actividades dentro y fuera del aula

- Enriquecimiento de las clases presenciales (López, 2007). 


\section{El constructivismo y las TIC}

El constructivismo es una teoría que propone que el ambiente de aprendizaje debesostener múltiples perspectivas o interpretaciones de realidad, construcción de conocimiento y actividades basadas en experiencias ricas en contexto. Esta teoría se centra en la construcción del conocimiento, no en su reproducción. Un componente importante del constructivismo es que la educación se enfoca en tareas auténticas, que son las que tienen una relevancia y utilidad en el mundo real (Hernández, 2008).

De esta manera, tenemos que el aprendizaje es un proceso constructivo, pero también es un proceso intencional, ya que supone el planteamiento de metas o propósitos por parte de los estudiantes. También es estratégico, puesto que requiere que los estudiantes apliquen métodos y utilicen recursos que les ayuden a asimilar significados, como las estrategias de organización de información o la elaboración de nuevos productos a partir de dicha información (Peñalosa, 2013).

Ausubel (1983) planteó que el aprendizaje del alumno depende de la estructura cognitiva previa que se relaciona con la nueva información, debe entenderse por "estructura cognitiva" al conjunto de conceptos e ideas que un individuo posee en un determinado campo del conocimiento, así como su organización.

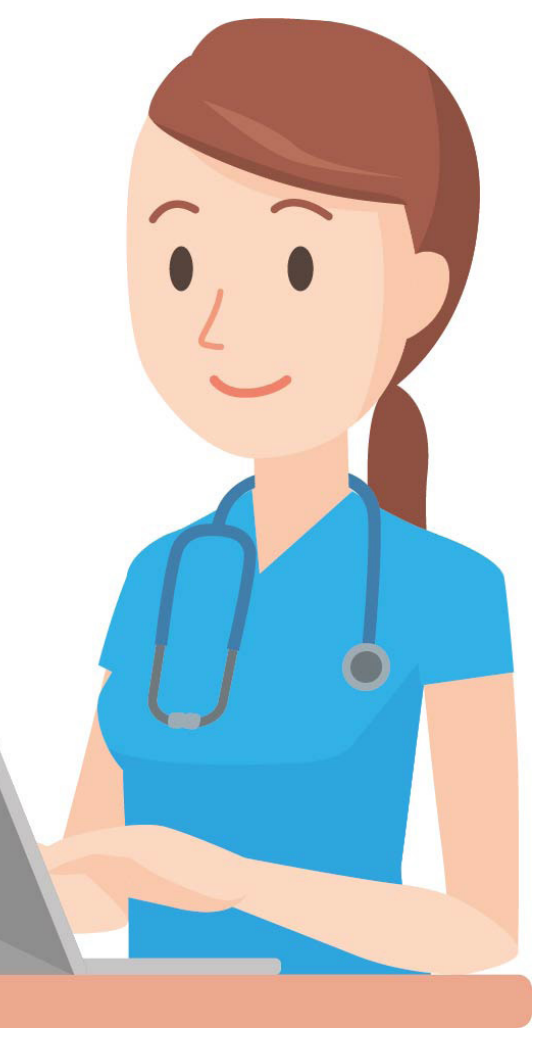

\section{Autonomía del estudiante en favor de su rendimiento académico}

El rendimiento académico es la correspondencia entre el comportamiento del alumno y los comportamientos institucionales especificados que se deben aprender en su momento escolar. Se trata de una relación tríadica entre lo que se pretende aprender, lo que facilita el aprendizaje y lo que se aprende (Passmore, 1983).

Lo que se aprende, corresponde a una dimensión político-social, ya que a partir de las políticas educativas se especifica lo que socialmente se considera adecuado que los estudiantes aprendan en cada nivel escolar. Lo que facilita el aprendizaje se refiere a una dimensión pedagógica, ya que incluye la elección e implementación de estrategias didácticas para facilitar el conocimiento. Lo que aprende el estudiante se centra en una dimensión psicológica porque considera el comportamiento del estudiante respecto a su objeto de estudio (Fuentes, 2004). 


\section{El uso de las TIC para el aprendizaje}

López de la Madrid (2007), en un estudio de caso observado en un periodo de tres años, detectó mejoría en el aprendizaje de los estudiantes al utilizar las Tıc.

Huertas y Pantoja (2016) en un estudio con 198 alumnos encontraron que la aplicación de un programa educativo basado en las TIc mejora el rendimiento escolar y la motivación del alumnado de forma significativa; sin embargo, y de acuerdo con Ávila (2014), la incursión de las tic en los ambientes académicos es un proceso que requiere una constante evaluación del impacto de estas herramientas, para optimizar el proceso principal de inclusión. En una revisión sistemática realizada por Lahti (2013) se encontró (en cuatro de sus estudios) la mejora asociada con el aprendizaje electrónico en comparación con las técnicas tradicionales de conocimiento, a pesar de que la diferencia no fue estadísticamente significativa.

En el área de la salud, también se ha explorado la implementación de las TIC para el aprendizaje de sus estudiantes. Maury (2014), en su estudio "Caracterización de la plataforma Moodle por estudiantes de ciencias de la salud", menciona que el uso de dicha plataforma motivó a los estudiantes a invertir tiempo en su aprendizaje, asumiendo esta actividad como parte de su rol profesional.

\section{Metodología}

Se comparó el rendimiento académico de 58 estudiantes antes y después de utilizar un blog como herramienta para mejorar su aprendizaje. Antes del uso del blog, se les aplicó un instrumento de exploración de conocimiento y uso de las Tic para el aprendizaje (UTAE-1). Asimismo, se determinaron algunas características de accesibilidad al recurso tecnológico desde sus hogares, el tiempo invertido en estas herramientas para favorecer el aprendizaje y la difusión de parte de los profesores para poder implementar las tıc en su proceso enseñanza-aprendizaje. El instrumento UTAE-1 fue creado, revisado por expertos en el área.

\section{Resultados}

De la muestra estudiada, los alumnos tenían entre 21 y 23 años. Se pudieron observar características como el preferir actividades grupales y el uso habitual y cotidiano de la tecnología, las cuales son características de una generación denominada por Ogg y Bonvalet como la generación del milenio (2006).

Se observó que 93\% de los estudiantes tiene acceso a internet desde su hogar (88\% de los estudiantes no lo hace desde la escuela). Su forma de conectarse a internet fuera del hogar es a través de redes abiertas (97\%) o uso 
de datos móviles (79.3\%), y 64\% de los estudiantes accesa a internet de 2 a 4 horas al día para realizar actividades escolares.

El 80\% tiene de 1 a 2 computadoras en su hogar ya sea portátil o de escritorio, 97\% cuenta con un celular inteligente (estos dos aparatos electrónicos son los que utilizan más frecuentemente para realizar actividades escolares) y $30 \%$ con una tableta o ipad; es decir, que $98 \%$ de los estudiantes cuenta con al menos un aparato electrónico en su casa.

Las TIC para el aprendizaje que fueron sugeridas por el profesor con más frecuencia son las revistas electrónicas.

A continuación, se muestra un cuadro correlacional del uso de las tıc y la dificultad presentada.

\begin{tabular}{|c|c|c|c|}
\hline \multicolumn{4}{|c|}{ Uso de TIC para el aprendizaje } \\
\hline \multicolumn{2}{|l|}{ Dificultad en TIC para el aprendizaje } & Sílo usa (\%) & No lo usa (\%) \\
\hline \multicolumn{4}{|l|}{ Foros de discusión } \\
\hline \multicolumn{2}{|l|}{ No tiene dificultad } & 3.2 & 96.8 \\
\hline \multicolumn{2}{|l|}{ Sí tiene dificultad } & 0.0 & 100.0 \\
\hline \multicolumn{4}{|l|}{ Blogs } \\
\hline \multicolumn{2}{|l|}{ No tiene dificultad } & 50.0 & 50.0 \\
\hline \multicolumn{2}{|l|}{ Sí tiene dificultad } & 50.0 & 50.0 \\
\hline \multicolumn{4}{|l|}{ Videoconferencias } \\
\hline \multicolumn{2}{|l|}{ No tiene dificultad } & 14.7 & 85.3 \\
\hline \multicolumn{2}{|l|}{ Sí tiene dificultad } & 0.0 & 100.0 \\
\hline \multicolumn{4}{|l|}{ Libros electrónicos } \\
\hline \multicolumn{2}{|l|}{ No tiene dificultad } & 76.1 & 23.9 \\
\hline \multicolumn{2}{|l|}{ Sí tiene dificultad } & 25.0 & 75.0 \\
\hline \multicolumn{4}{|l|}{ Revistas electrónicas } \\
\hline \multicolumn{2}{|l|}{ No tiene dificultad } & 27.1 & \\
\hline & 40.0 & 60.0 & \\
\hline \multicolumn{3}{|c|}{ Creador de representaciones visuales } & \\
\hline No tiene dificultad & 93.0 & 7.0 & \\
\hline Sí tiene dificultad & 0.0 & 100.0 & \\
\hline $\mathrm{n}=58$ & & & \\
\hline
\end{tabular}

Los estudiantes afirman tener dificultad en las tic como videoconferencias y, por tanto, no utilizarlas. Por otra parte, los libros electrónicos, revistas electrónicas y creadores de presentaciones visuales son las TIC en las que los estudiantes no presentan dificultad para utilizarlas en su aprendizaje.

Las herramientas más utilizadas fueron previamente sugeridas por el profesor para realizar sus actividades escolares. Los blogs no tuvieron un 
resultado significativo, por lo que no se pudo realizar una correlación entre su uso y dificultad.

Se realizó una prueba estadística para evaluar el rendimiento académico de los estudiantes, tomando en cuenta sus calificaciones antes y después de usar el blog como herramienta auxiliar en el aprendizaje; el rendimiento no presentó cambios relevantes. Un estudiante con altas calificaciones, no siempre es el que tiene mayores habilidades, capacidades o aptitudes. Para poder aprender de manera significativa, se necesita tener interés sobre la temática a instruirse y conocimiento previo al implementar una Tıc que no será usada para socializar, sino para optimizar el tiempo de estudio.

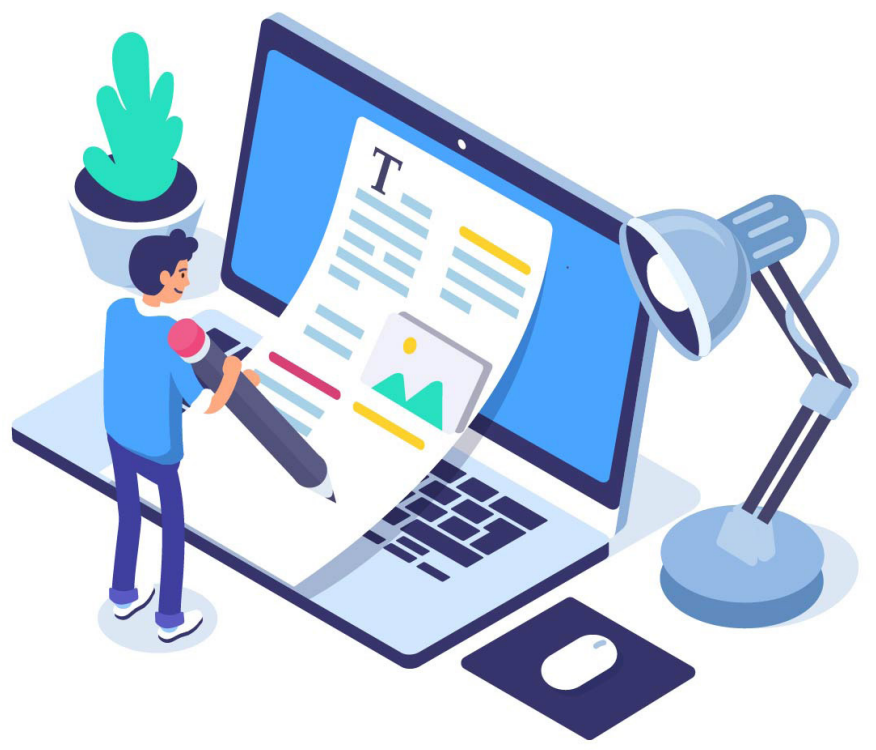

El indicador del nivel educativo adquirido en los países desarrollados y en vías de desarrollo ha sido, sigue y probablemente seguirá siendo las calificaciones escolares, ya que son reflejo de la evaluación donde el alumno demuestra sus conocimientos sobre las distintas áreas que el sistema considera necesarias para su desarrollo como miembro activo de la sociedad (Edel, 2003).

El aumento en el uso de las TIC por parte de los estudiantes no es aislado, debe orientarse hacia un mejor aprovechamiento de sus capacidades de aprendizaje (López, 2013). Si el uso de las tecnologías es frecuente y éstas se expanden como formas de entretenimiento es probable que estimulen el ensayo y error, y la respuesta rápida, (Litwin, 2005); lo que nos lleva a creer que su uso cotidiano propicia ciertas habilidades de pensamiento en los estudiantes, que agilizan su capacidad de comprensión de los contenidos y aseguran un aprendizaje más significativo.

Por otra parte, se observó que los estudiantes expuestos al uso del blog como herramienta de apoyo para el aprendizaje, durante su práctica clínica, tenían una actitud positiva previa ante el uso de la tecnología, lo que contribuyó a una mejor aceptación por parte de los estudiantes para utilizarlas (86\% consideró que el uso de equipos electrónicos y el acceso a internet favorecerían su aprendizaje).

Hernández (2008) menciona que el nivel de dominio que tengan los estudiantes se relaciona con su actitud. En su estudio observó que quienes declararon no dominar alguna herramienta tecnológica presentaron una actitud neutral ante el uso de las TIC, contra quienes se dijeron ser expertos ante el uso de una herramienta tecnológica, pues mostraron tener una muy buena actitud. En este mismo estudio, aquellos estudiantes con una mejor actitud declararon que $60 \%$ de su actividad estudiantil implicaba el uso de recursos tecnológicos. 
La diversidad de las nuevas tecnologías es muy amplia, por lo que es importante saber seleccionar el medio a utilizar con los estudiantes, su manejo, sus aspectos pedagógicos, formas de aplicación y los aspectos fuertes y débiles de la tıc a utilizar.

Aún cuando las calificaciones no reflejen un cambio superior esperado con el uso de una herramienta extra en el aprendizaje, el estudiante puede optimizar y mejorar sus estrategias dentro del ámbito escolar con el uso de las TIC y obtener mayor conocimiento.

En la profesión de enfermería, así como en cualquier otra profesión, el tiempo invertido para el aprendizaje es de suma importancia, de ello depende el conocimiento actualizado y asegura una mejor praxis de los procedimientos a realizar en escenarios reales. Al tener un mundo de información nacional e internacional de temáticas relacionadas con las ciencias de la salud, los momentos docente-alumno dentro del aula son insuficientes para poder lograr la transmisión de todo aquello que se requiere para mantenerse a la vanguardia. Es por ello que en nuestro actual siglo los avances tecnológicos deben ser aprovechados por ambos entes (alumno y docente) para ir en búsqueda de un apoyo en las formas de enseñar y aprender.

\section{Conclusiones}

Las TIC para el aprendizaje, las herramientas tecnológicas y el acceso a internet de manera gradual, han influenciado al ser humano y han modificado la forma de comunicarse, informarse, actualizarse, socializar y también de educarse. Como todos los cambios a través de la historia, se necesita de la evaluación y de la inclusión de estas nuevas formas de enseñanza a las metodologías convencionales.

Godoy-Rodríguez (2006) mencionó que el efecto del uso de las tic sobre los logros académicos puede ser muy diferente en estudiantes inmersos en un ambiente rico en tecnología que sobre aquellos en entornos comunes o poco innovadores. Hay que recordar que el aprendizaje de un alumno va más allá de sus calificaciones, que requiere de elementos internos -capacidades y habilidades previas que como persona se pueden utilizar a nivel académico para superarse - y externas — ambientes de aprendizaje, métodos de enseñanza entre profesores, innovación y vigencia de los conocimientos a transmitir-.

En la actualidad, enseñar la profesión de enfermería, requiere de un amplio criterio de innovación, en donde el enfoque educativo sea multicéntrico. El objetivo es hacer participe al alumno y lograr su autogestión para construir un profesional crítico, capaz de asimilar, crear y aplicar sus conocimientos en escenarios reales. 
La universidad debe situarse más allá de un entorno convencional, debe ser el lugar en donde se generen los conocimientos necesarios para crecer, alcanzar metas y objetivos. Los docentes son partícipes activos de este proceso $y$, en trabajo conjunto con estudiantes, autoridades e instituciones, deben incluir las herramientas necesarias para mejorar los ambientes de aprendizaje, ya sea en momentos teóricos o prácticos.

\section{Referencias}

- Ausubel, N. H. (1983). Psicología educativa. Un punto de vista cognoscitivo (2a ed.). México: Trillas.

* Avila-Fajardo, G. P. y Riascos-Erazo S. C. (2011). Propuesta para la medición del impacto de las tic en la enseñanza universitaria, 14(1), 169-188.

* Instituto Nacional de Estadística y Geografía (INEGI) (2011). Disponibilidad y uso de tecnología de información y comunicaciones en los Hogares. Recuperado de: https:// www.inegi.org.mx/programas/modutih/2011/.

* Díaz, F. y Hernández G. (2010). Estrategias docentes para un aprendizaje significativo. Una interpretación. México: McGraw Hill.

* Edel, N. R. (2016). El rendimiento académico; concepto, investigación y desarrollo. Revista Iberoamericana sobre Calidad, Eficacia y Cambio en Educación, 1(2), 1-15. Recuperado de: https://www.redalyc.org/pdf/551/55110208.pdf.

* Fuentes, N. T. (2004). El estudiante como sujeto del rendimiento académico. Sinéctica, agosto 2004 - enero 2005, 23-27. Recuperado de: https://sinectica. iteso.mx/index.php/SINECTICA/article/view/265/258.

- García, A. (2005). De la educación a distancia a una educación sin distancia. México: Bened.

* Godoy, R. C. E. (2006). Usos educativos de las TIC: competencias tecnológicas y rendimiento académico de los estudiantes universitarios barineses, una perspectiva causal. Educere, 10(35), octubre-diciembre, 661-70. Recuperado de: http://www.redalyc.org/pdf/356/35603512.pdf.

* Hernández, R. S. (2008). El modelo constructivista con las nuevas tecnologías: aplicado en el proceso de aprendizaje. RUSC. Universities and Knowledge Society Journal, 5(2), 26-35. Dol: http://dx.doi.org/10.7238/rusc.v5i2.335.

* Huertas Montes, A. y Pantoja Vallejo, A. (2016). Efectos de un programa educativo basado en el uso de las TIC sobre el rendimiento académico y la motivación del alumnado en la asignatura de tecnología de educación secundaria. UNED, 19(2), 229-250. DOI: https://doi.org/10.5944/educxx1.16464.

- Lahti, M., Hätönen, H. y Välimäki, M. (2014). Impact of e-learning on nurses and student nurses knowledge, skills, and satisfaction: A systematic review and metaanalysis. International Journal of Nursing Studies, 51(1), 136-149. Dol: https://www. doi.org/10.1016/j.ijnurstu.2012.12.017. 
- Ley, K. y Young, D. B. (2015). Instructional Principles for self-regulation. Educational Technology Research and Development, 49(2), 93-103. Dol: https://doi.org/10.1007/ BF02504930.Litwin, E. (2005). Tecnologías educativas en tiempos de internet. Argentina: Amorrortu.

- López Carrasco, M. A. (2013). Los medios o recursos que utilizar: la presencia de las TIC. En Aprendizaje, competencias y TIC (p.). México: Pearson Educación.

* López Castañeda, E., Ferreiro Martínez, V. V, Brito Laredo J. y Garambullo, A. I. (2011). La generación del milenio, su diversidad y sus implicaciones en el desarrollo académico. Revista internacional administracion \& finanzas, 4(1), 67-82. Recuperdado de: ftp://ftp.repec.org/opt/ReDIF/RePEc/ibf/riafin/riaf-v4n1-2011/ RIAF-V4N1-2011-5.pdf.

* Gargallo López, B., Suárez Rodríguez, J. y Ferreras Remesal, A. (2007). Estrategias de aprendizaje y rendimiento académico en estudiantes universitarios. Revista de Investigación Educativa, 25 (2), 421-441. Recuperado de: http://www.redalyc.org/ pdf/2833/283321923010.pdf.

* Maury-Sintjago, E., Pereira- Centurión, T., Labbé-Gibert M., Sepúlveda P. y Valdebenito, C. (2015). Caracterización del uso de La plataforma MOODLE por estudiantes de ciencias de la salud. Edumecentro, 7(1), 4-17. Recuperado de: http:// scielo.sld.cu/pdf/edu/v7n1/edu02115.pdf.

* Ogg, J. \& Bonvalet, C. (2006). The baby-boomer generation and the birth cohort of 1945-1954; a European perspective. Recuperado de: https://youngfoundation. org/wp-content/uploads/2013/06/03 06 Europeanbabyboomers.pdf.

* Passmore, J. (1983). Filosofía y enseñanza. México: Fondo de Cultura Económica.

* Peñalosa, C. E. (2013). Fundamentos para el aprovechamiento docente de tecnologías de la información y la comunicación: un modelo. En Peñalosa C. E. (Ed.), Estrategias docentes con tecnologías: guía práctica (pp.1-5). México: Pearson.

* Wison, J. D. (1995). Como valorar la calidad de la enseñanza. Madrid: Paidós.

\section{Cómo citar este artículo}

* López Colmenares, Guadalupe Nohemí (2019). Aplicando las TIC en el aprendizaje de la enfermería profesional. Revista Digital Universitaria (RDU). Vol. 20, núm. 5 septiembre-octubre. Dol: http://doi.org/10.22201/codeic.16076079e.2019.v20n5. a8.

Recepción: 15/02/2019. Aprobación: 06/07/2019 